\title{
Reliability Impact of Dynamic Thermal Rating System in Wind Power Integrated Network
}

\author{
Jiashen Teh and Ian Cotton, Senior Member, IEEE
}

\begin{abstract}
Electrical networks across the globe are gearing towards environmentally friendly operation with many renewable sources being introduced. Among them, the popularity of electrical generation by wind has gained the most ground. Wind, as one of the meteorological conditions, also has high influence on line ratings and such manner of operating the line is known as dynamic thermal rating (DTR) system. Seeing this relationship, it is intuitively important to investigate the reliability of a electrical network incorporating DTR and wind farm. Hence, the objective of this paper is to propose a methodology that can perform this task. In the methodology, sequential Monte Carlo (SMC) simulation was performed due to time dependencies of line ratings and wind power. As a result, modeling of these time-series data was performed using the auto-regressive and moving-average (ARMA) model. Moreover, correlations between line rating and wind power were also considered to provide a practical approach to the modeling. Results have shown that DTR system is able to increase network reliability and allows for higher wind energy penetration.
\end{abstract}

Index Terms - Dynamic thermal rating, Monte Carlo, reliability, smart grid.

\section{INTRODUCTION}

D YNAMIC thermal rating (DTR) system is part of a larger suite of smart grid technologies that allows electrical conductors to operate at higher capacities based on their local weather conditions. It does this by increasing the conductors' thermal ratings while adhering to the maximum operating design temperatures [1], [2]. In particular, the DTR technique applicable to a bare overhead conductor has been formulated in the IEEE standard 738 [3] and is widely accepted in the industry [4]. In it, the steady-state transmission line ratings are calculated as a function of wind speed $\left(V_{w}\right)$, wind angle $(\phi)$, air temperature $\left(T_{a}\right)$, solar radiation angle $(\theta)$, conductor resistance $(R)$, and its operating temperature $\left(T_{c}\right)$, as shown in (1):

$$
Q_{c}\left(T_{c}, T_{a}, V_{w}, \phi\right)+Q_{r}\left(T_{c}, T_{a}\right)=Q_{s}(\theta)+I^{2} R\left(T_{c}\right)
$$

Manuscript received April 07, 2015; revised August 10, 2015; accepted October 17, 2015. This work was supported in part by Universiti Sains Malaysia and in part by Ministry of Higher Education, Malaysia. Associate Editor: E. Pohl.

J. Teh is with the School of Electrical and Electronics Engineering, Universiti Sains Malaysia, Penang, Malaysia, and also with the School of Electrical and Electronics Engineering, University of Manchester, Manchester, U.K. (e-mail: jiashen.teh-2@manchester.ac.uk).

I. Cotton is with the School of Electrical and Electronics Engineering, University of Manchester, Manchester, U.K. (e-mail: ian.cotton@manchester.ac.uk).

Color versions of one or more of the figures in this paper are available online at http://ieeexplore.ieee.org.

Digital Object Identifier 10.1109/TR.2015.2495173 where $Q_{c}, Q_{r}$, and $Q_{s}$ are the convection heat loss, radiated heat loss, and solar heat gain, respectively, calculated as a function of their weather elements. $R$ is not a constant and is affected by the conductor temperature. Detail mathematical descriptions of these elements are not given in this paper due to space limitation and prospective readers are referred to [3]. Finally, $I$ is the conductor current capacity in unit Ampere and is determined by rearranging (1). This current capacity is also known as the line thermal rating.

In a DTR system, the real-time values of weather elements are measured by sensors and they are used to periodically update the line ratings - - hence the dynamic behavior. As the actual weather conditions are most of the time more desirable than their conservative assumed values, DTR line ratings do not underestimate the actual ratings and allow full utilization of the line capacity [5]. Consequently, it has been reported that DTR system is able to increase the transmission line rating by $10 \%$ to $30 \%$ over $90 \%$ of the time with improvement of $50 \%$ being possible in windy areas [6], [7]. This frees up unused line capacities and reduces the needs for new conductors. Besides that, monitoring of line ratings also provide operators with network status conditions.

However, due to under-development of sensory technology in the past, the majority of transmission lines were historically given a fix rating based on a set of conservative weather assumptions, also known as the static thermal rating (STR) [8]. As a result, transmission capacity in the past easily bottlenecked due to underestimation of line capacity, leading to many constructions of new transmission corridors. Despite a non-issue in the past, this option is less relevant today due to scarcity of land and space. To make things worse, electrical demand will continue to rise into the future. In light of that, DTR system is bound to gradually replace STR system and become more commonplace.

The ability of a DTR system in enhancing the power system energy transfer capacity has inspired wide varieties of studies. First, the field applications of a DTR system have proven its reliability benefits in transmission networks [9], [10]. The reliability of a DTR system and its impact on the reliability of a power network was evaluated [11]. Transmission line rating prediction model up to a day ahead based on DTR capacity was developed [12]. Apart from transmission network, DTR system was shown to improve the reliability of a distribution network as well [13]. DTR system has also been used to provide greater flexibility in load shedding strategy as line congestions are mitigated and power flows are more easily redirected [14]. Finally, due to the worldwide successful deployment of wind farms [15], DTR system was shown to facilitate the integration of wind energy [16]-[18]. This is possible as faster wind velocity produces 
more wind energy and higher line rating at the same time [19]. For this reason, the recent emphasis of energy sector on renewables sources has been placed on wind power [20], [21].

Apart from DTR, a large part of the successful wind farm operations is also contributed by its well-understood reliability characteristics. For example, due to expensive wind farm operational and maintenance $(\mathrm{O} \& \mathrm{M})$ cost, a model that provides practical O\&M guidelines to reduce repair cost and increase the marketability of wind energy was proposed [22]. Besides that, a decision support model based on option theory was proposed to maximize the remaining useful life of wind turbine while minimizing failure risk [23]. Lastly, a method able to estimate the wind turbine component health condition based on online information collected about their observable lives was proposed [24]. From power system reliability viewpoint, the reliability impacts of wind farm in power system generation adequacy were investigated by several models [25]-[28]. The synergy of energy storage and wind farm was captured in [25]. A detailed Markov model based on frequency and duration approach was used to model wind farm generation adequacy in [26]. A probabilistic approach was used to conduct the reliability impact of wind farm generation adequacy in [28]. In these studies, little to no attention was given towards the reliability of transmission adequacy. Other than the reliability impact of wind energy on power system, the reliability modeling of wind farm components was performed as well [29]. The paper reported aspects that must be considered when modeling offshore wind farms. A different approach based on universal generating functions was also used to set up the reliability model of wind farms [30].

Despite the advantage of incorporating DTR system in a wind farm operation, none of the cited work above has jointly performed a power system reliability study consisting of the two. Hence, this paper set out to investigate the reliability impact of DTR system in a wind powered electrical network and a novel methodology for doing that was proposed. The methodology presented in this paper takes on the planning perspective and the real-time constraint of DTR system application is not considered.

\section{Composite Reliability Evaluation}

Reliability evaluation of power system has been a major discussion and has generated much research interest [31], [32]. Fundamentally, it is performed in three layers [33]. The first layer is concerned with the adequacy assessment of power system, where only the ability of generation to satisfy the load is studied. The second layer expands the former layer by incorporating the transmission network. Reliability evaluation at this stage is affected by the transmission network capacity and is also known as the composite reliability evaluation. Finally, the third layer adds on more complexity by considering the distribution network. Normally, this layer is not performed as it is very time consuming and complicated [33]. In this paper, as the DTR system is studied on a transmission network and wind farms are incorporated, the reliability evaluations were performed on the composite level.

Power systems are normally large and consist of many components. The possible combinations of components statuses can quickly escalate into an unmanageable amount of data. On top of that, solving optimum power flows (OPF) in a power network is highly nonlinear. Hence, if OPFs were performed on each combination, the process would take up an indefinite amount of time to complete. For this simple reason, analytical method using the enumeration technique is unsuitable in power system reliability studies. On the other hand, Monte Carlo (MC) simulation offers a simple way to explore this huge amount of state space by running it for a large number of times [34].

There are two types of MC simulation-sequential (SMC) and non-sequential MC (NSMC) [35]. In NSMC simulation, each power system components is assigned a probability distribution and statuses of each component are sampled randomly. The power system state is obtained by consolidating all component states and the reliability of the power network is evaluated. In NSMC, the chronological events of the component statuses are ignored. Contrary to that, SMC considers the time dependency factor but at a greater computational cost. Other than that, they are both the same. In this paper, the SMC simulation is performed and the explanation is given next.

Wind farms converts wind speed kinetic energy into electrical energy. Thus, the modeling of wind speed and wind turbine energy curve for the conversion of wind speed to wind power is essential [36]. According to [15], wind speed is not entirely random and is more appropriately described using the time-series auto-regressive and moving-average (ARMA) model. This is contrary to the popular distribution fit that has no time element. Moreover, ARMA can generate time-series wind speed well beyond its original data used during ARMA model fitting and actual wind speed trend can be simulated. Due to the chronological nature of wind speed ARMA model, the converted wind powers are also time based. This chronological behavior extends to all weather parameters used to calculate the line ratings and consequently the line rating itself. In light of the time-series wind powers and line ratings, it is evident that the application of DTR systems and wind farms for power system reliability evaluation should be performed using the SMC simulations.

There are various indices that can be used to describe the composite reliability evaluation of power system [33], [37]. Among which loss of load expectation (LOLE) shows the average duration of inability to satisfy electrical demand and loss of energy expectation (LOEE) depicts the average amount of load loss. Although both can be used, only LOEE is used in this paper as it takes the longest time to converge [33]. Hence, the convergence of LOEE ensures that adequate MC simulations have been performed and the resulted expected reliability index is closest to the true value. Besides that, a new index term designated as the expected wind power delivered (EWPD) is created in this paper to describe the average delivery of wind power through the transmission network.

\section{Methodology}

In this section, the proposed methodology used to evaluate the reliability impact of DTR system in a wind generated power network is described. 


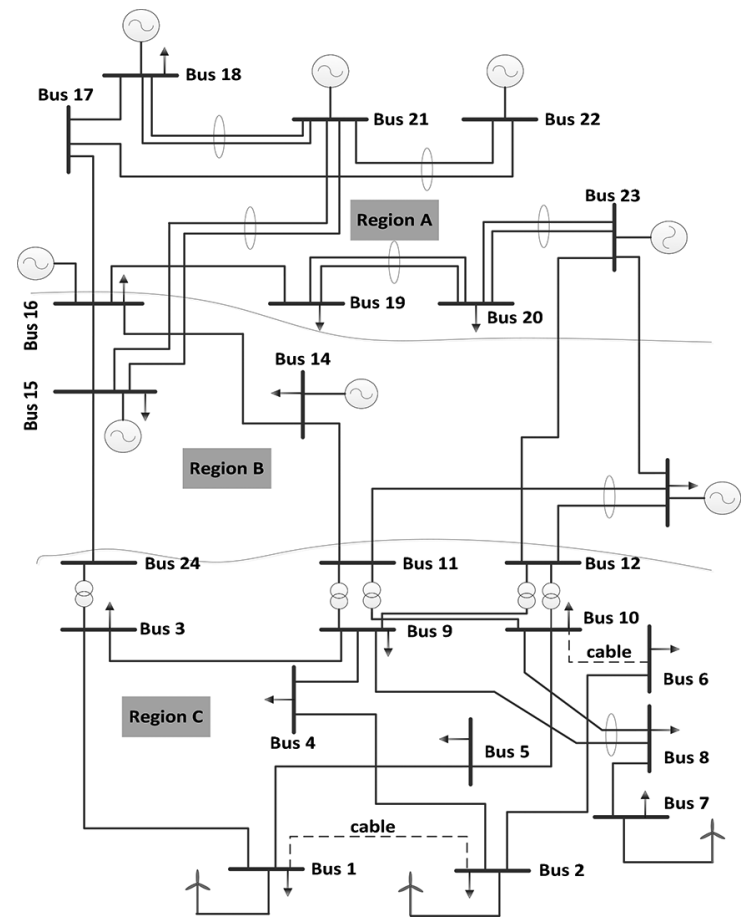

Fig. 1. Modified IEEE 24-bus reliability test network.

\section{A. Reliability Test Network}

In the realm of reliability studies, IEEE 24-bus Reliability Test Network (RTN) is widely utilized [38]. Abiding to the norm, this paper also utilized the RTN with slight modifications as shown in Fig. 1 where the proposed methodology is tested.

As can be seen, the RTN is divided into three regions notated as region A, B, and C. It was assumed that the distance between regions is increasing in distance. For example, region $\mathrm{C}$ is further away from region $\mathrm{A}$ than region $\mathrm{B}$ is. Notice that in region $\mathrm{C}, 3$ of its conventional generators were replaced with wind farms. Each line of the RTN is also given a DTR system. Special cases such as lines with common tower and common right-of-way share the same DTR system as they are closed together. The test network and its modifications are shown in Fig. 1.

\section{B. Transmission Line Reliability Model}

The RTN network provides the reliability data (i.e., failure and repair rates) for all the lines. These values are considered constant; hence, an underlying exponential distribution is assumed. Utilizing the values, a simple 2-state Markov model (MM) is proposed to represent the transition of the line between the up and down states. As SMC simulation is performed, random values of mean-time-to-failure (MTTF) and mean-time-to-repair (MTTR) of the lines are used to describe this transition. During the simulations, exponential random variate MTTFs and MTTRs are obtained by converting uniformly generated random numbers using the inverse transform method to represent their underlying distributions [35]. Due to the exponentially distributed failure and repair rates of the lines, the conversions are performed as in (2):

$$
\begin{aligned}
\text { MTTF } & =-\frac{1}{\lambda} \ln U \\
\text { MTTR } & =-\frac{1}{\mu} \ln U
\end{aligned}
$$

where $U$ is the uniform random number, $\lambda$ is the failure rate, and $\mu$ is the repair rate.

\section{DTR System Reliability Model}

In order for successful application of DTR technology, the DTR system needs to be functional. Hence, it is also important to quantify its reliability and incorporate it into the proposed methodology. It is well known that DTR system relies on its sensors to sample parameters that influence the conductor thermal ratings, transmitter, and receiver for data communication back and forth with the control center and data bandwidth for communication [39]. For simplicity, it is assumed that all communication devices and bandwidth are always available. Besides that, individual modeling of the DTR sensors are not performed as it is not the focus of this paper. Rather, a simple 2-state MM is used to describe the DTR system. It is considered that the DTR system has failure and repair rate of 3/year and 364/year, respectively. Depending on the installed technologies, the failure and repair rate of the DTR system may vary. Lastly, the DTR system MTTF and MTTR can be determined using (1) as well.

\section{Line Rating and Wind Speed ARMA Model}

After considering the common tower and common right-of-way lines, it was counted that there are 21 DTR systems in the RTN. Hence, 21 historical line ratings are needed. Similarly, historical wind speeds from 3 sites are needed for the 3 wind farms modeled in the test network.

Following that, weather parameters (according to IEEE Standard 738) required for line ratings calculations from 21 sites and wind speeds needed for wind power calculations from 3 sites were sampled from the British Atmospheric Data Center (BADC) website [40]. In all of the samplings, hourly values for 1 historical year were used. Moreover, weather parameters in each region of the RTN were sampled from sites that are close to each other, no further than $50 \mathrm{~km}$ apart. On the other hand, weather parameters in between the regions were sampled from sites that are about $100 \mathrm{~km}$ apart.

All ARMA models were fitted using the educational software ARMASA [41]. Generally, an ARMA model with $n$-order autoregressive and $m$-order moving average $(n, m)$ is as shown in

$$
\begin{array}{r}
y_{t}=\alpha_{1} y_{t-1}+\alpha_{2} y_{t-2}+\cdots+\alpha_{n} y_{t-n}+e_{t}-\beta_{1} e_{t-1} \\
-\beta_{2} e_{t-2}-\cdots-\beta_{m} e_{t-m}
\end{array}
$$

where $\alpha_{i}(i=1,2, \ldots, n)$ and $\beta_{j}(j=1,2, \ldots, m)$ are the auto-regressive and moving-average constants of the model, respectively. $e_{t}$ is a normal white noise process with zero mean and a variance of $\sigma^{2}-e_{t}$ is normally and independently distributed $\left(e_{t} \in \operatorname{NID}\left(0, \sigma^{2}\right)\right)$. Equation (3) shows that a conditional mean value of $y_{t}$ in a future time $t$ from a sample containing all $y$ is dependent on its past observed values $y_{t-i}(i=$ 
$1,2, \ldots, n)$ and past innovations $e_{t-j}(j=1,2, \ldots, m)$. In our case, $y_{t}$ is representing either wind speeds or line ratings.

\section{E. Wind Farm Model}

Wind farm is a wind energy conversion system (WECS) that consist of two parts - the natural kinetic energy of wind speed and wind turbines (WTs) [42]. As all WTs are subjected to the same wind at an instance, the amount of electrical output depends on the number of functional WTs which are affected by their reliabilities. In turn, the reliabilities of WTs are affected by their capacities, locations, designs, access logistic, and maintenance regimes [43]. Hence, the determination of a realistic reliability value for wind turbines is case specific.

Due to that, a reliability survey on only five of the current most popular WT designs was performed by [44]. The rated power of the considered WTs was also set to $5 \mathrm{MW}$ to reflect the development in the current wind industry. The survey sourced its studies from the Windstats survey in Denmark and Germany, Schleswig Holstein survey in Germany, and the statistics in Sweden, which are all long-term operational records of WTs at various installation sites. Due to the trustworthiness of these surveys, the average statistical reliability data of WT derived by [44] is adopted in this paper. As the reliability values of five WT designs were derived with minute differences, this paper uses only the value from the most reliable WT design as found in [44, Table 9, column 5]. That is, a WT design based on directly driven permanent magnet synchronous generator (DDPMSG). The failure and repair rate of DDPMSG wind turbine are 1.501/year and 0.0081/year, respectively. This decision is further justified by studies in [42] and [45] that have shown the variability in the reliability of a single WT has no significant impact on the power network reliability indices. For further simplicity, all WTs are assumed to be the same and share the same reliability data.

Next, the relationship between wind speed and WT electrical output used in this paper is described. Based on the model developed in [46], the power output of a WT unit expressed as a function of wind speed is shown in (4):

$$
P\left(V_{w}\right)= \begin{cases}0 & 0 \leq V_{w}<V_{c i} \\ \left(A+B V_{w}+C V_{w}^{2}\right) P_{r} & V_{c i} \leq V_{w}<V_{r} \\ P_{r} & V_{r} \leq V_{w}<V_{c o} \\ 0 & V_{w} \geq V_{c o}\end{cases}
$$

where $V_{w}$ is the wind velocity and $P_{r}$ is the rated power output of the wind turbine. $V_{c i}, V_{r}$, and $V_{c o}$ is the cut-in, rated, and cut-out wind speed, respectively. The piecewise linear equation of (3) is also known as the wind power curve and is illustrated in Fig. 2 for visualization. Finally, the constant $A, B$, and $C$ are calculated as (5):

$$
\begin{aligned}
& A=\frac{1}{\left(V_{c i}-V_{r}\right)^{2}}\left[V_{c i}\left(V_{c i}+V_{r}\right)-4\left(V_{c i} V_{r}\right)\left(\frac{V_{c i}+V_{r}}{2 V_{r}}\right)^{3}\right] \\
& B=\frac{1}{\left(V_{c i}-V_{r}\right)^{2}}\left[4\left(V_{c i} V_{r}\right)\left(\frac{V_{c i}+V_{r}}{2 V_{r}}\right)^{3}-\left(3 V_{c i}+V_{r}\right)\right] \\
& C=\frac{1}{\left(V_{c i}-V_{r}\right)^{2}}\left[2-4\left(\frac{V_{c i}+V_{r}}{2 V_{r}}\right)^{3}\right]
\end{aligned}
$$

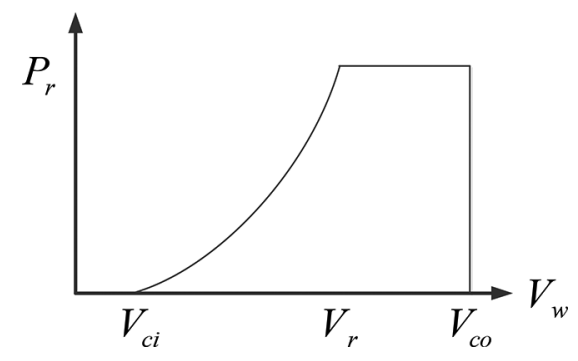

Fig. 2. Wind power curve of WTG unit.

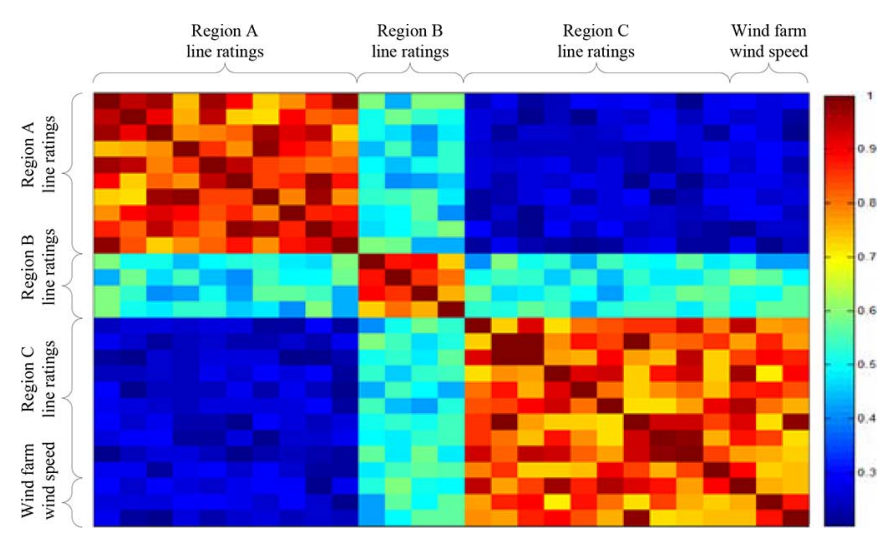

Fig. 3. Correlation among historical line ratings in region A, B, C and wind speeds in 3 wind farm.

\section{F. Line Rating and Wind Speed Correlation Model}

Conductors and wind farms that are geographically located near to each other are affected by strongly correlated weather conditions. This correlation diminishes in strength as they are separated further apart. As the ARMA models were fitted according to their respective historical data, the correlations among the historical data are also inherited into the ARMA models. Such relationship should be reflected in the simulations of the ARMA model so that the generated time-series values are also correlated in the same manner.

To do that, the correlations of the ARMA models need to be evaluated first and the Pearson's product-moment as stated in (6) was selected to quantify the correlations. The notation "cov" is the covariance between any two vectors and $\sigma$ is the standard deviation:

$$
\rho_{x, y}=\frac{\operatorname{COV}(x, y)}{\sigma_{x} \sigma_{y}} .
$$

Following that, the correlation of historical line ratings and wind speeds of three wind farms were determined and the results are shown in Fig. 3. It shows that the correlations are strong within each region as the weather parameters were sampled from sites that are close to each other (within $50 \mathrm{~km}$ ). The correlation reduces when the regions were compared against each other due to larger separation between the sites where the weather data was sampled. As expected, the correlation is the weakest when the regions are furthest apart, i.e., region $\mathrm{A}$ and C.

The simulations of the ARMA models are dependent on its random innovations that are sampled from a normal distribution 
as mentioned earlier in Section III-D. Hence, by generating vectors of correlated random seeds for all ARMA models, the simulated time-series values are also correlated in the same manner. To do that, the univariate normal distributions were generalized into its equivalent multivariate format before the random seeds were sampled. This multivariate normal distribution is stated in (7):

$$
f(x)=\frac{1}{(2 \pi)^{p / 2}|\Sigma|^{1 / 2}} \exp \left\{-\frac{1}{2}(x-\mu)^{\mathrm{T}} \Sigma^{-1}(x-\mu)\right\}
$$

The value $p$ is the number of ARMA model. $\mu$ is a vector of mean value of the random seeds of all ARMA models. In this case, it is the zero vectors as the innovations of all ARMA models have zero mean error. Finally, $\Sigma$ is the symmetric covariance matrix of all ARMA models and it can be obtained by simple rearrangement of (6). As the covariance matrix is specified by the correlation matrix, the randomly generated seeds and consequently the simulated time-series values are also correlated in the same way.

Alternatively, the random seeds can be generated using the Cholesky decomposition [47], eigenvalue decomposition, or genetic algorithm [42].

\section{G. Evaluation Procedure}

Classical reliability index such as LOEE has been used to measure the composite reliability evaluations carry out in this paper. Besides that, a self-proposed index coined as the expected wind power delivered (EWPD) is also used to describe the average wind power delivered. In respect to SMC simulation performance in this paper, the LOEE and EWPD are determined using (8) and (9), respectively:

$$
\begin{aligned}
\text { LOEE } & =\frac{1}{N} \sum_{s=1}^{N} \sum_{h=1}^{8760} \mathrm{LNS}_{h}(\mathrm{MWh} / \mathrm{yr}) \\
\mathrm{EWPD} & =\frac{1}{N} \sum_{s=1}^{N} \sum_{h=1}^{8760} \mathrm{WP}_{h}(\mathrm{MWh} / \mathrm{yr})
\end{aligned}
$$

where $\mathrm{LNS}_{h}$ is the load not served at hour $h, s$ is the sample of the simulation, and $N$ is the total number of sample. $\mathrm{WP}_{h}$ is the wind power delivered at hour $h$. Finally, the procedure for evaluating the reliability impact of DTR system in the wind powered IEEE 24-bus RTN is described:

1) Fit the historical line ratings and wind speeds from 3 wind farm sites using the ARMA models.

2) Generate the hourly DTR ratings for all lines and wind speeds for 3 wind farm sites according to their correlation relationships.

3) Convert the wind speeds into wind power output according to the wind power curve.

4) Generate the up-down cycle of transmission lines, generators, and DTR systems.

5) Input the hourly DTR ratings, wind power, line, and DTR system statuses into the RTN.

6) Perform SMC simulation and record the reliability indices.

7) Repeat the steps above from 2) to 6) for $N$ amount of samples until convergence of LOEE is met. That is, its coefficient of variation drops below $5 \%$.

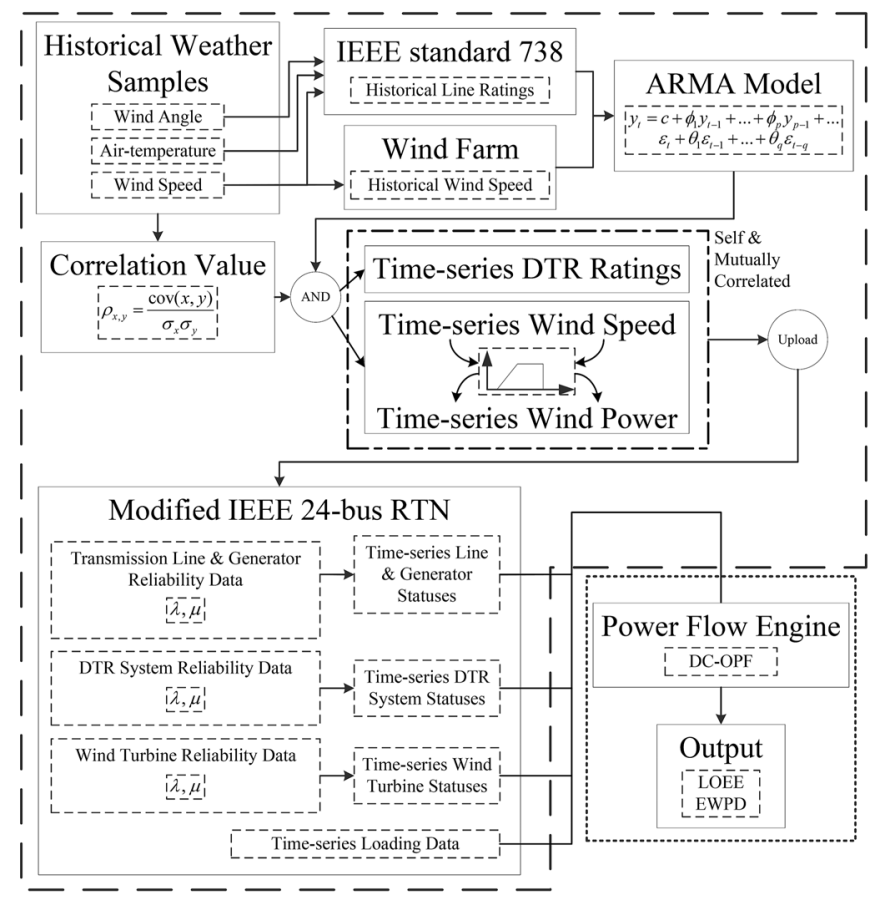

Fig. 4. Overview of the proposed methodology.

The given descriptions depicting the procedures and overview of the methodology is also in accordance with the illustration shown in Fig. 4.

\section{Results}

The main objective of this paper is to demonstrate a practical methodology for evaluating the reliability impact of implementing DTR system in a wind power electrical network. The rest of this section systematically laid out the results obtained.

\section{A. Base Case Reliability Evaluation}

The first case study was performed in the RTN by incrementally increasing its loading level by $20 \%$ up to until twice the original loading level. LOEE and EWPD of the network with and without DTR system were compared as shown in Fig. 5(a) and (b), respectively. The case without DTR is also known as Static case.

In Fig. 5(a), the results show that at low loading level, Static case performed slightly better than the DTR. The reason is because at low loading levels, both the rating methods are able to meet the electrical demands. On top of that, there were some instances where the DTR fell below the STR. Hence, during low loading periods, STR gives the perception that it is better than DTR and it is sufficient for the network reliability. However, as the load increases, the LOEE of both cases quickly diverge and the DTR has substantially lower LOEE than the Static case. This is very much due to the ability of DTR to use its rating enhancement to damp the effect of line outages which in the Static case were not available. Consequently, Static case requires more load shedding in absence of other corrective actions in place.

In Fig. 5(b), it shows that the EWPD of DTR case is always higher than the Static case. In other words, the rating enhancements given by the DTR allows for more utilization wind power 
(a)

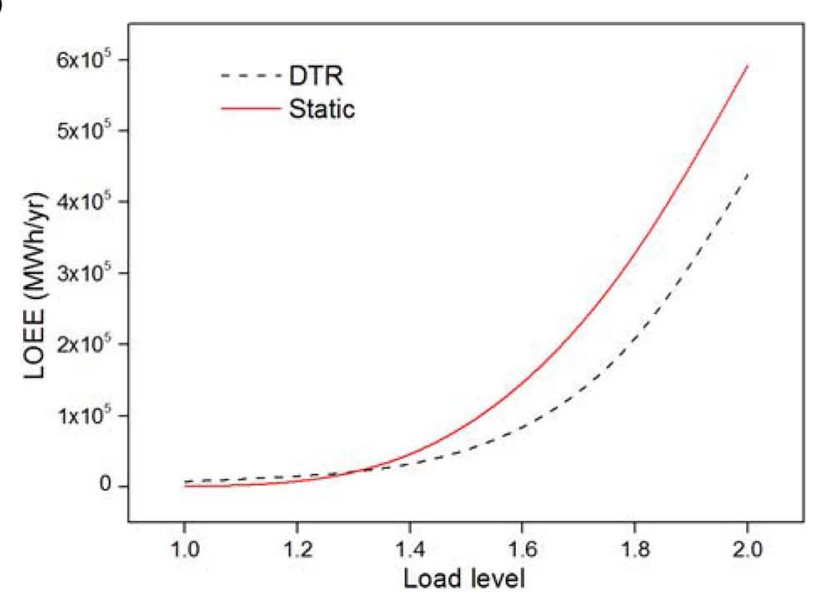

(b)

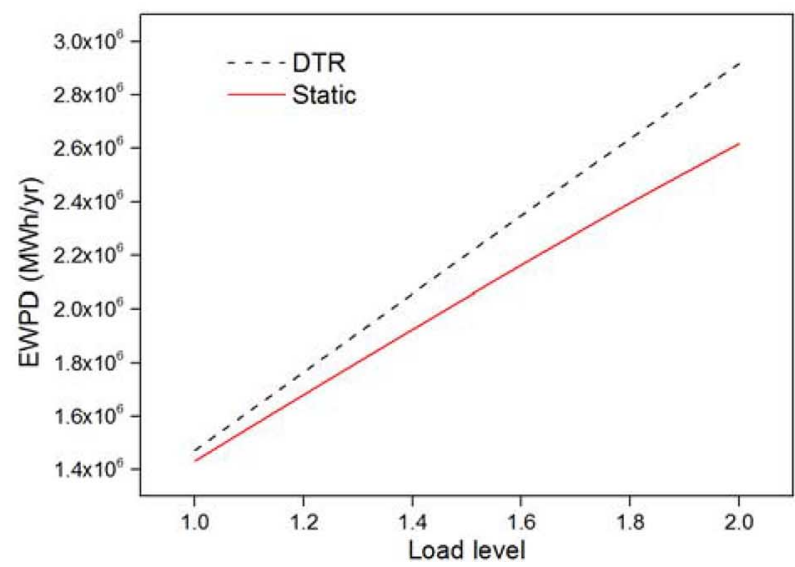

Fig. 5. (a) LOEE and (b) EWPD of DTR and Static case across various loading levels.

generated in the network. This is made possible by considering the high correlation between the DTR ratings and wind speed of the wind farm sites. That is, high wind power outputs are coupled with high line ratings. In the Static case however, its low rating assumption is the main cause of low wind power penetration instead of the correlation effect mentioned previously.

\section{B. Correlation Effect}

Thus far, the proposed methodology was implemented by considering the cross and self-correlations between line ratings and wind speeds. This section intends to investigate the effect of varying this correlation relationship.

Fig. 6(a) shows the result of LOEE at three correlation levels in the DTR case across the same loading levels as in section A. The "Base Case" is the scenario where the original correlations were maintained. "Uncorrelated" and "Totally Correlated" are scenarios where the correlation relationships were made zero and one, respectively. The results show that at low loading level, correlation has only little effect towards the LOEE. However, as the loading increases, the Uncorrelated scenario consistently has lower LOEE, signifying an underestimation of the reliability index. On the other hand, the Totally Correlated scenario quickly increase above the Base Case at 1.8 loading level and beyond, signifying an overestimation of the reliability index. (a)

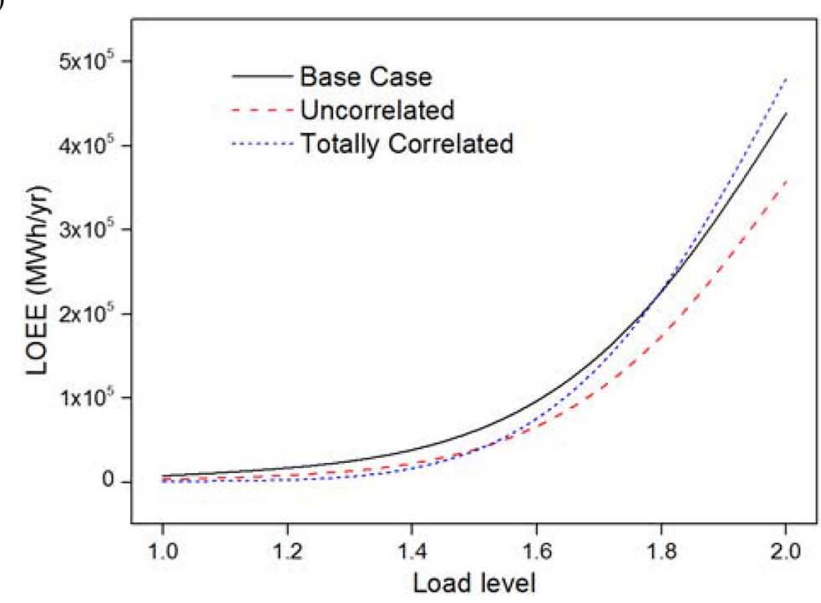

(b)

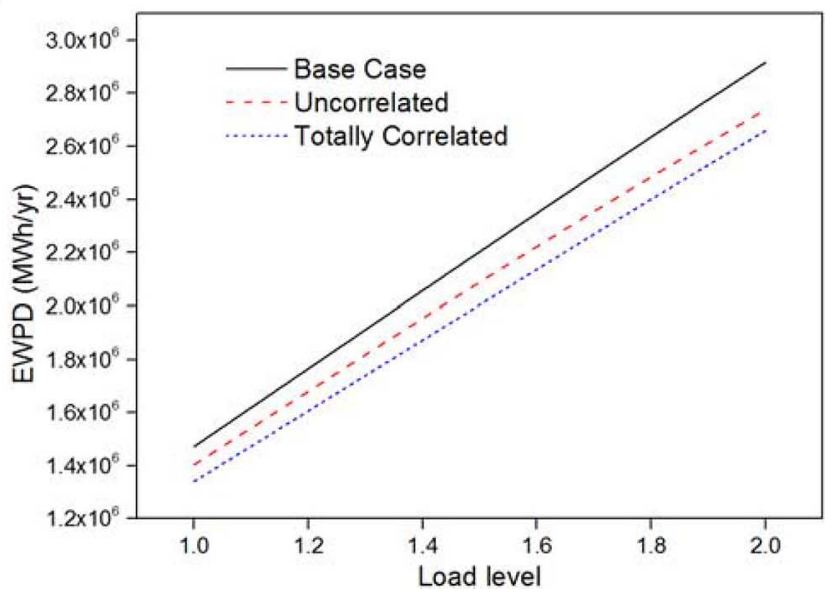

Fig. 6. (a) LOEE and (b) EWPD of DTR case at different correlation levels across various loading levels.

Under the same conditions, Fig. 6(b) shows the effect of correlation towards the EWPD index. It shows that when actual correlation levels were not considered, the EWPD index is always underestimated. The effect is most pronounced under the Totally Correlated scenario and the negative effect is lesser under the Uncorrelated scenario.

\section{DTR System Reliability Effect}

The reliability of DTR system was also varied to determine its impact on the RTN reliability. Four cases of DTR system reliability were studied by cutting and enhancing its reliability by $80 \%$ and $40 \%$ and the effects on the LOEE and EWPD are shown in Fig. 7(a) and (b), respectively. The results show that in general, the RTN reliability indices are not sensitive towards the reliability of the DTR system. None the less, the effect is emphasized as the loading increases. The less reliable DTR system produces higher LOEE and lower EWPD as it has higher failure durations.

\section{Wind Power Penetration Effect}

Finally, the wind power penetration levels were investigated to determine the impact of DTR system towards the reliability of RTN. LOEE of the system was recorded and was compared 
(a)

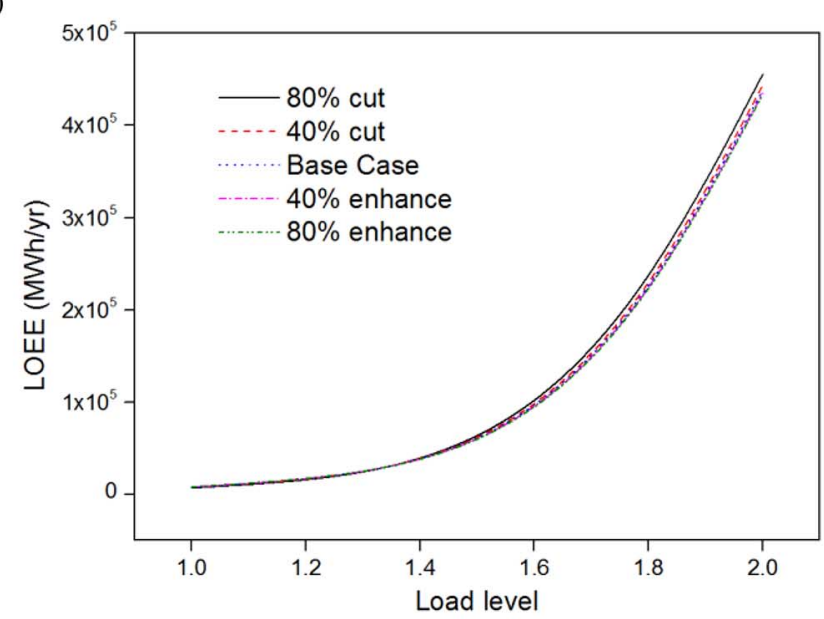

(b)

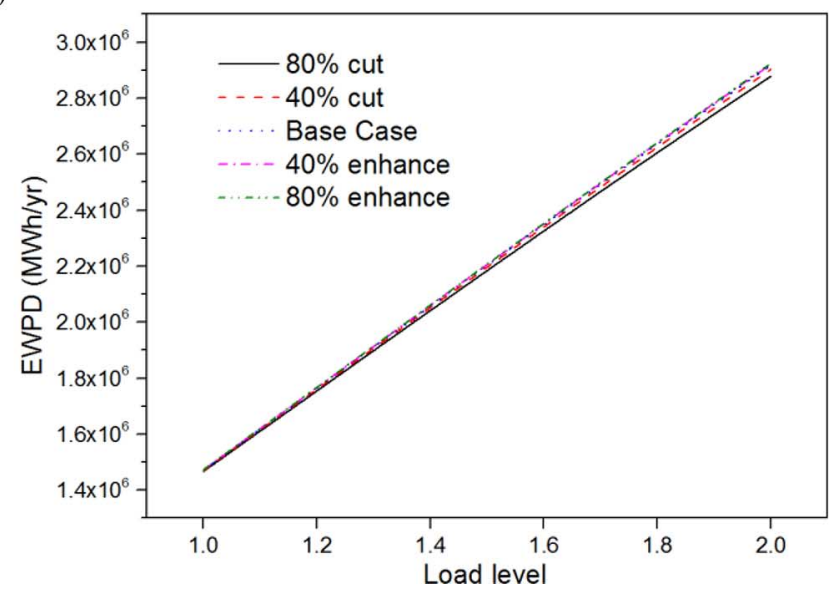

Fig. 7. (a) LOEE and (b) EWPD of different DTR system reliabilities across various loading levels.

with the Static case. The investigations were performed by increasing the load level twice the original value with base case DTR system and the results are shown in Fig. 8. It shows that DTR system can accommodate much larger wind power than its static counterpart across all wind power penetration levels. This proves that the DTR system is able to effectively receive high wind power integration into the network.

\section{Discussion}

\section{A. Adequacy of DTR System}

All results show that the DTR system is able to reduce load loss when compare to the STR system. However, the presented results are only from the effect of DTR enhancement. From the practical perspective, the level of LOEE presented is not acceptable by the industrial practice, especially during high load level. Therefore, as part of a holistic approach to smart grid integration, the DTR system can couple with demand side management (DSM), energy storage, or even flexible AC transmission (FACT) devices to drive up the reliability of the network.

For example, DSM can be used to shift more loads to meet with high rating period or arrange low loading conditions with low rating periods. When DTR ratings are high and wind is strong, excessive wind power generated can be stored in energy

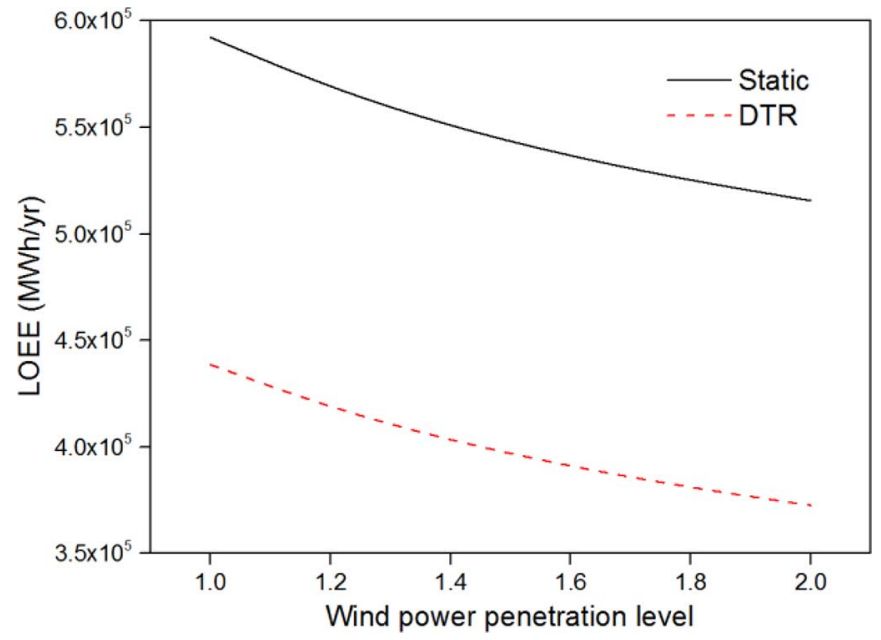

Fig. 8. LOEE DTR and Static case across various wind penetration levels at loading level twice the original amount.

storage and can be used to help satisfy loads during high energy demands. Moreover, if the energy given by energy storage is insufficient, DSM can be used to further flatten the loading profiles. On the other hand, FACTS devices are used to stabilize voltage profile of buses and provide reactive power supplementation to drive up the transmission of real power.

\section{B. Financial Benefit of DTR System and Wind Farm}

Sustainable living and environmental conservation policy calls for replacement of fossil fuel generator with renewable energy based generator. Wind energy is considered one of the most popular renewables and has developed rapidly in the past decade. Besides low to no impact on environment conditions, wind farm also needs no burning fuel. Hence, the generation cost is virtually free. Although, wind generation is hard to control, large scale implementations with other renewables are able to replace conventional generation completely. For example, Costa Rica has managed to replace $100 \%$ of their electrical generation with renewables energy for the first 75 days of year 2015 [48].

DTR system also has huge financial implications to the network. For example, a scheme implemented by Scottish Power Network in the U.K. shows that implementing DTR cost $10 \%$ lesser than network reinforcement would require. It is foreseeable from economies of scale that wide implementation of DTR system can further drive down the cost. Current and classical networks are operated with redundancy. Such manner of asset management is stable and deterministic but very expensive and inefficient in terms of asset utilization. In comparison to this, new technologies such as DTR system would fare better if the same cost-benefit analysis was performed. For example, the results in this paper show that DTR system produces better reliability indices than STR system without introducing additional infrastructures.

\section{CONCLUSION}

This paper presents a novel methodology for assessing the impact of DTR system on the reliability of wind powered network. The studies show that networks with low demands do 
not benefit from DTR system. However, at high loading levels, DTR system can help to reduce the amount the load shedding required to achieve a balance system. Without the presence of DTR system, new lines would be needed for alleviating the stress on the network. In other words, DTR system enables higher operational risk without needing new infrastructures that would otherwise cause system instability in the classical electrical network.

It is by no means that this paper advocates only DTR system. Instead, DTR system is part of the larger selections of smart grid technologies. By integrating DTR system with other new technologies such as DSM, energy storage, etc., future electrical networks are able to operate much closer to their security limit while safely adhering to components safety designs. In other words, the reliability of electrical network is increased.

The analysis of this paper takes into account the time series behavior of wind and line ratings. Through this, practical simulations of wind speeds and line ratings are achieved. Besides that, the correlation between line ratings and wind speeds of the wind farms are also taken into consideration. Inclusion of this relationship acknowledges the mutual influence of the time-series behavior which is also reflected in their natural weather parameters.

Finally, this paper proves that DTR systems do make a significant contribution to the power system reliability. Nonetheless, the current network planning and expansion methodology does not take this technology into account. To be fair, other promising smart grid technologies such as DSM and energy storage are not inspected as well. Hence, in order for full potential of DTR system to be unlocked effectively, working standards and regulations need to be updated and improved while classical networks are continually moving towards the smart grid paradigm.

\section{REFERENCES}

[1] J. F. Hall and A. K. Deb, "Economic evaluation of dynamic thermal rating by adaptive forecasting," IEEE Trans. Power Del., vol. 3, pp. 2048-2055, 1988

[2] M. W. Davis, "A new thermal rating approach: The real time thermal rating system for strategic overhead conductor transmission lines-Part I: General description and justification of the real time thermal rating system," IEEE Trans. Power App. Syst., vol. PAS-96, pp. 803-809, 1977.

[3] IEEE Standard for Calculating the Current-Temperature of Bare Overhead Conductors, IEEE Std. 738-2006 (Revision of IEEE Std 738-1993), 2007, pp. c1-59.

[4] D. M. Greenwood, J. P. Gentle, K. S. Myers, P. J. Davison, I. J. West, J. W. Bush, G. L. Ingram, and M. C. M. Troffaes, "A comparison of real-time thermal rating systems in the U.S. and the U.K," IEEE Trans. Power Del., vol. 29, pp. 1849-1858, 2014.

[5] Guidelines for Increased Utilization of Existing Overhead Transmission Lines, CIGRE Working Group B2.13 2008.

[6] Guidelines for Increased Utilization of Existing Overhead Transmission Line, 2008, CIGRE Brochure.

[7] O. Seppa, M. Clements, S. Damsgaard-Mikkelsen, R. Payne, and N. Coad, "Application of real time thermal ratings for optimizing transmission line investment and operating decisions," CIGRE Paper, pp. 22-301, 2000

[8] "Guide for the selection of weather parameters for bare overhead conductor ratings," Cigre Brochure 2006.

[9] D. A. Douglass, A. Edris, and G. A. Pritchard, "Field application of a dynamic thermal circuit rating method," IEEE Trans. Power Del., vol. 12, pp. 823-831, 1997.

[10] D. A. Douglass and A. Edris, "Real-time monitoring and dynamic thermal rating of power transmission circuits," IEEE Trans. Power Del., vol. 11, pp. 1407-1418, 1996.
[11] H. Shaker, H. Zareipour, and M. Fotuhi-Firuzabad, "Reliability modeling of dynamic thermal rating," IEEE Trans. Power Del., vol. 28, pp. 1600-1609, 2013.

[12] W. J. Steeley, B. L. Norris, and A. K. Deb, "Ambient temperature corrected dynamic transmission line ratings at two PG\&E locations," IEEE Trans. Power Del., vol. 6, pp. 1234-1242, 1991.

[13] A. Safdarian, M. Z. Degefa, M. Fotuhi-Firuzabad, and M. Lehtonen, "Benefits of real-time monitoring to distribution systems: Dynamic thermal rating," IEEE Trans. Smart Grid, vol. 6, pp. 2023-2031, 2015.

[14] S. Wei-Qing, Z. Yan, W. Cheng-Min, and S. Ping, "Flexible load shedding strategy considering real-time dynamic thermal line rating," IET Gene., Transm., Distrib., vol. 7, pp. 130-137, 2013.

[15] R. Billinton, H. Chen, and R. Ghajar, "Time-series models for reliability evaluation of power systems including wind energy," Microelectron. Rel., vol. 36, pp. 1253-1261, 9, 1996.

[16] A. K. Kazerooni, J. Mutale, M. Perry, S. Venkatesan, and D. Morrice, "Dynamic thermal rating application to facilitate wind energy integration," in Proc. IEEE Trondheim PowerTech., 2011, pp. 1-7.

[17] J. Heckenbergerova and J. Hosek, "Dynamic thermal rating of power transmission lines related to wind energy integration," in Proc. 11th Int. Conf. Environment and Electrical Engineering (EEEIC), 2012, pp. 798-801.

[18] T. Ringelband, M. Lange, M. Dietrich, and H. J. Haubrich, "Potential of improved wind integration by dynamic thermal rating of overhead lines," in Proc. 2009 IEEE Bucharest PowerTech, 2009, pp. 1-5.

[19] J. W. Jerrell, W. Z. Black, and T. J. Parker, "Critical span analysis of overhead conductors," IEEE Trans. Power Del., vol. 3, pp. 1942-1950, 1988.

[20] H. Bevrani, A. Ghosh, and G. Ledwich, "Renewable energy sources and frequency regulation: Survey and new perspectives," IET Renew. Power Gener., vol. 4, pp. 438-457, 2010.

[21] Second Progress Report on the Promotion and Use of Energy From Renewable Sources for the United Kingdom, European Commission, 2013.

[22] B. Eunshin, L. Ntaimo, and D. Yu, "Optimal maintenance strategies for wind turbine systems under stochastic weather conditions," IEEE Trans. Rel., vol. 59, pp. 393-404, 2010.

[23] G. Haddad, P. A. Sandborn, and M. G. Pecht, "An options approach for decision support of systems with prognostic capabilities," IEEE Trans. Rel., vol. 61, pp. 872-883, 2012.

[24] R. J. de Andrade Vieira and M. A. Sanz-Bobi, "Failure risk indicators for a maintenance model based on observable life of industrial components with an application to wind turbines," IEEE Trans. Rel., vol. 62, pp. 569-582, 2013.

[25] P. Hu, R. Karki, and R. Billinton, "Reliability evaluation of generating systems containing wind power and energy storage," IET Gener., Transm., Distrib., vol. 3, pp. 783-791, 2009.

[26] A. S. Dobakhshari and M. Fotuhi-Firuzabad, "A reliability model of large wind farms for power system adequacy studies," IEEE Trans. Energy Convers., vol. 24, pp. 792-801, 2009.

[27] R. Karki, H. Po, and R. Billinton, "A simplified wind power generation model for reliability evaluation," IEEE Trans. Energy Convers., vol. 21, pp. 533-540, 2006.

[28] Z. Yi, A. A. Chowdhury, and D. O. Koval, "Probabilistic wind energy modeling in electric generation system reliability assessment," IEEE Trans. Ind. Applicat., vol. 47, pp. 1507-1514, 2011.

[29] N. B. Negra, O. Holmstrom, B. Bak-Jensen, and P. Sorensen, "Aspects of relevance in offshore wind farm reliability assessment," IEEE Trans. Energy Convers., vol. 22, pp. 159-166, 2007.

[30] A. R. Di Fazio and M. Russo, "Wind farm modelling for reliability assessment," IET Renew. Power Gener., vol. 2, pp. 239-248, 2008.

[31] "Bibliography on the application of probability methods in power system reliability evaluation 1971-1977," IEEE Trans. Power App. Syst.., vol. PAS-97, pp. 2235-2242, 1978.

[32] R. Billinton, M. Fotuhi-Firuzabad, and L. Bertling, "Bibliography on the application of probability methods in power system reliability evaluation 1996-1999," IEEE Power Eng. Rev., vol. 21, pp. 56-56, 2001.

[33] R. Billinton and R. N. Allan, Reliability Evaluation of Power Systems. New York, NY, USA: Plenum, 1984.

[34] W. Li, Risk Assessment of Power System. Piscataway, NJ, USA IEEE-Wiley, 2005.

[35] R. Billinton and W. Li, Reliability Assessment of Electric Power Systems Using Monte Carlo. New York, NY, USA: Plenum, 1994.

[36] F. D. Pullen and D. J. Hopgood, "Probabilistic approaches to transmission line thermal ratings," in Proc. 3rd Int. Conf. Probabilistic Methods Applied to Electric Power Systems, 1991, pp. 191-196. 
[37] A. K. David, "Reliability criteria for power systems with inherent generation and transmission deficiencies," IEEE Trans. Rel., vol. R-30, pp. $18-21,1981$.

[38] P. M. Subcommittee, "IEEE reliability test system," IEEE Trans. Power App. Syst., vol. PAS-98, pp. 2047-2054, 1979.

[39] Y. Yi, F. Lambert, and D. Divan, "A survey on technologies for implementing sensor networks for power delivery systems," in Proc. IEEE Power Eng. Soc. General Meeting, 2007, pp. 1-8.

[40] B. A. D. C. (BADC) [Online]. Available: http://badc.nerc.ac.uk/home/

[41] P. M. T. Broersen, "Automatic spectral analysis with time series models," IEEE Trans. Instrum. Meas., vol. 51, pp. 211-216, 2002.

[42] Y. Gao and R. Billinton, "Adequacy assessment of generating systems containing wind power considering wind speed correlation," IET Renew. Power Gener., vol. 3, pp. 217-226, 2009.

[43] F. Spinato, P. J. Tavner, G. J. W. van Bussel, and E. Koutoulakos, "Reliability of wind turbine subassemblies," IET Renew. Power Gener., vol. 3, pp. 387-401, 2009.

[44] Q. Gao, C. Liu, B. Xie, and X. Cai, "Evaluation of the mainstream wind turbine concepts considering their reliabilities," IET Renew. Power Gener., vol. 6, pp. 348-357, 2012.

[45] R. Billinton and G. Yi, "Multistate wind energy conversion system models for adequacy assessment of generating systems incorporating wind energy," IEEE Trans. Energy Convers., vol. 23, pp. 163-170, 2008.

[46] P. Giorsetto and K. F. Utsurogi, "Development of a new procedure for reliability modeling of wind turbine generators," IEEE Power Eng. Rev., vol. PER-3, pp. 38-38, 1983.
[47] R. L. Iman and W. J. Conover, "A distribution-free approach to inducing rank correlation among input variables," Commun. Statist.-Simulat. Computat., vol. 11, pp. 311-334.

[48] R. Spencer, "Costa Rica has only used renewable energy this year," The Telegraph UK, 2015.

Jiashen Teh is currently working toward the Ph.D. degree at the University of Manchester, Manchester, U.K.

His research interests include modeling of transmission line thermal ratings and reliability studies of future transmission system.

Ian Cotton (SM'07) received the Ph.D. degree in electrical engineering from the University of Manchester Institute of Science and Technology, Manchester, U.K, in 1998 .

He is currently a Professor with the Electrical Energy and Power Systems Group, University of Manchester. His current research interests include power systems transients, high voltage systems application in aerospace, and power system-induced corrosion. He is also the director of the Manchester Energy Group. 